\title{
GEOMETRIA DO SINCLINAL GANDARELA BASEADA NA DECONVOLUÇÃO EULER 2D E 3D - QUADRILÁTERO FERRÍFERO (MG)
}

\author{
Natália Valadares de Oliveira ${ }^{1}$ Issamu Endo² e Luiz Gabriel Souza de Oliveira ${ }^{3}$ \\ Recebido em 04 abril, 2005 / Aceito em 20 outubro, 2005 \\ Received on April 04, 2005 / Accepted on October 20, 2005
}

\begin{abstract}
Euler deconvolution, based on the Euler's equation, is a powerful tool that can be applied in the estimative of mean depths of magnetic sources or magnetic bodies. This procedure, combined with geological and structural data, showed the definition of the geometry in depth of the Gandarela syncline region, located in the center-north portion of the Quadrilátero Ferrífero, Minas Gerais. Its structural framework is defined as a reclined fold with NE-SW trending axial trace and hinge zone plunging to the southeast. The Gandarela syncline is composed of thick metasedimentary units of Minas Supergroup and of greenstone sequence of the Rio das Velhas Supergroup. The results based on 2D and 3D Euler deconvolution applied in magnetic data from Brasil-Alemanha aerogeophysical project allowed to estimate the mean depths of the magnetic sources probably, associated to iron formations (Cauê Formation and Nova Lima Group). Additionally, the geometry of the structure was confirmed by $2 \mathrm{D}$ magnetic modeling.
\end{abstract}

Keywords: 2D-3D Euler Deconvolution, aeromagnetic data, Gandarela Syncline.

RESUMO. A deconvolução Euler, baseada na equação homogênea de Euler, é uma técnica poderosa que pode ser utilizada na estimativa de profundidades médias de fontes magnéticas ou de corpos magnéticos. Este procedimento, aliado a interpretação de dados geológico-estruturais, possibilitou a caracterização da geometria em profundidade da região do Sinclinal Gandarela, localizado na porção centro-norte do Quadrilátero Ferrífero. Sua estruturação é definida como uma dobra reclinada cujo traço axial se orienta segundo a direção NE-SW e a charneira, localizada na sua porção meridional, apresenta caimento moderado para SE. Em termos litológicos, é formado por metassedimentos pertencentes ao Supergrupo Minas, estando em contato com as seqüências vulcano-sedimentares do Supergrupo Rio das Velhas e as rochas que compõem o embasamento arqueano (Complexo Metamórfico Caeté). Os resultados alcançados a partir da deconvolução de Euler 2D e 3D aplicada em dados magnetométricos levantados pelo projeto aerogeofísico Brasil-Alemanha permitiram estimar as profundidades médias de fontes magnéticas possivelmente associadas às formações ferríferas (Formação Cauê e Grupo Nova Lima). Permitiu também caracterizar a morfologia da estrutura sendo confirmada por modelagens magnetométricas $2 \mathrm{D}$ executadas na região.

Palavras-chave: Deconvolução Euler 2D e 3D, Aeromagnetometria, Quadrilátero Ferrífero, Sinclinal Gandarela.

\footnotetext{
${ }_{1}^{1}$ PPG-ECRN - Departamento de Geologia/Escola de Minas/Universidade Federal de Ouro Preto, Campus Morro do Cruzeiro s/n, 35400-000 Ouro Preto, Minas Gerais, Brasil. Tel: (31) 3559-1605 - E-mail: natalia@degeo.ufop.br

2PPG-ECRN - Departamento de Geologia/Escola de Minas/Universidade Federal de Ouro Preto, Campus Morro do Cruzeiro s/n, 35400-000 Ouro Preto, Minas Gerais, Brasil. Tel: (31) 3559-1605 - E-mail: issamu@degeo.ufop.br

${ }^{3}$ Departamento de Física - Instituto de Ciências Exatas e Biológicas/Universidade Federal de Ouro Preto, PPG-ECRN - Departamento de Geologia/Escola de Minas, Universidade Federal de Ouro Preto, Campus Morro do Cruzeiro s/n, 35400-000 Ouro Preto, Minas Gerais, Brasil. Tel: (31) 3559-1676 - E-mail: luizgabriel@degeo.ufop.br
} 


\section{INTRODUÇ̃̃̃O}

A região do Quadrilátero Ferrífero (QFe), embora muito conhecida e estudada, apresenta 0 arcabouço geológico e a evolução tectônica complexos, resultando em modelos tectônicos polifásicos (e.g. Dossin et al., 1992; Chauvet \& Menezes, 1992; Chemale et al., 1991; Chemale et al., 1994; Endo, 1997; Alkmim \& Marshak, 1998; Hippertt \& Davis, 2000), possivelmente pela falta de informações valiosas que a geofísica pode fornecer sobre a estrutura da crosta profunda.

São escassos os estudos geofísicos na região QFe e adjacências, sendo que os primeiros trabalhos datam no final da década de 70. Gasparini et al. (1979), através do estudo da superfície Curie na região, realizaram estimativas sobre as variações na composição química crustal. Blitzkow et al. (1979) realizaram modelagens gravimétricas bidimensionais, estimando a espessura crustal da região.

Padilha (1982) dedicou-se às unidades do Supergrupo Minas visando à inversão 2D de dados magnetométricos na região do sinclinal Gandarela. Utilizando-se da formação ferrífera Cauê como camada-guia e combinando diversas técnicas de filtragens, suprimiu das anomalias principais os efeitos perturbadores de anomalias vizinhas de interesse secundário, possibilitando inferir e fazer estimativas sobre as dimensões dos corpos causadores dessas anomalias.

Tavares (1998) utilizou dados gamaespectrométricos, magnetométricos, geológicos e estruturais para o estudo das potencialidades auríferas e da evolução estrutural do greenstone belt na região de Caeté. Silva (1999) investigou o greenstone belt Rio das Velhas no intuito prever áreas favoráveis a mineralizações auríferas via modelos estatísticos aplicados aos dados geofísicos.

Rolim (2001) aplicou os dados magnetométricos, gamaespectrométricos e eletromagnéticos do Projeto Rio das VeIhas/Convênio Brasil-Alemanha, para a exploração de ouro em terrenos do tipo greenstone belt Rio das Velhas.

Oliveira (2005) caracterizou a geometria do sinclinal Gandarela e do homoclinal Curral (QFe), baseada na integração de dados geológicos e geofísicos (Projeto Brasil-Alemanha) através de técnicas que auxiliam na identificação e no ajuste de profundidades de fontes magnéticas como a deconvolução Euler 2D, método poligonal 2D e inversões Linear e não-Linear 2D.

Com 0 desenvolvimento de técnicas computacionais aplicadas a dados magnetométricos, a interpretação, elaboração de modelos geológicos e as explorações de hidrocarbonetos e minerais tornaram-se mais confiáveis. 0 trabalho clássico de Peters (1949) e a técnica de deconvolução de Werner, originalmente proposta por Werner (1953) e desenvolvida por Hartman et al. (1971), impulsionaram a adaptação e criação de novas ferramentas para estimar profundidades de fontes causadoras de anomalias magnéticas, como a técnica de deconvolução Euler proposta por Hood (1965), posteriormente, desenvolvida por Thompson (1982), Durrheim \& Cooper (1998) em duas dimensões e, implementada por Reid et al. (1990) para três dimensões.

0 objetivo principal do presente trabalho é estimar as profundidades médias do embasamento onde o sinclinal Gandarela se encontra inserido e delimitar a sua peculiar geometria, através da deconvolução Euler 2D e 3D.

\section{CONTEXTO GEOLÓGICO}

0 sinclinal Gandarela está localizado na porção norte do Quadrilátero Ferrífero (Fig. 1), e é formado por metassedimentos do Supergrupo Minas em contato com o Grupo Nova Lima, pertencente ao Supergrupo Rio das Velhas e o embasamento composto por rochas graníticas do Complexo Metamórfico Caeté. É considerado hoje como uma região com grandes perspectivas para prospecção de minério de ferro (e. g. Endo \& Rocha Filho, 2003).

Dorr (1969) esboçou um dos primeiros modelos tectônicos para 0 sinclinal Gandarela e caracterizou a geometria como uma megadobra com fechamento para SE, em profundidade, com eixo orientado segundo a direção NE-SW (Fig. 2a). Padilha (1982) caracterizou a geometria do sinclinal Gandarela através da inversão não-linear 2D de dados magnetométricos do Projeto Brasil-Alemanha (DNPM, 1974), onde a Formação Ferrífera Cauê foi utilizada como camada-guia, concluindo que as estruturas tendem a se fechar em profundidade, mergulhando para sudeste (Fig. 2b).

Os estudos conduzidos por Franco (2003) no sinclinal Ouro Fino, situado a sul do sinclinal Gandarela, mudaram de forma radical a visão geométrica sobre essa estrutura. 0 modelo atual do sinclinal Ouro Fino é uma dobra reclinada com eixo de caimento moderado para ESE, com falso fechamento em profundidade (Fig. 2C). Como já verificado por Endo \& Rocha Filho (2003), o sinclinal Gandarela e o sinclinal Ouro Fino eram conectados e, posteriormente desconectados pela falha do Fundão ( $0^{\prime}$ Rourke, 1957; Maxwel, 1960), apresentando as mesmas características geométricas do sinclinal Ouro Fino.

Endo et al. (2004) conceberam um modelo para região Gandarela como um sinclinal antifórmico, resultante de três fases deformacionais. A primeira fase D1 resultou numa dobra recumbente com o Supergrupo Rio das Velhas no interior da nucleação da dobra, a fase D2 proporcionou um redobramento do flanco 


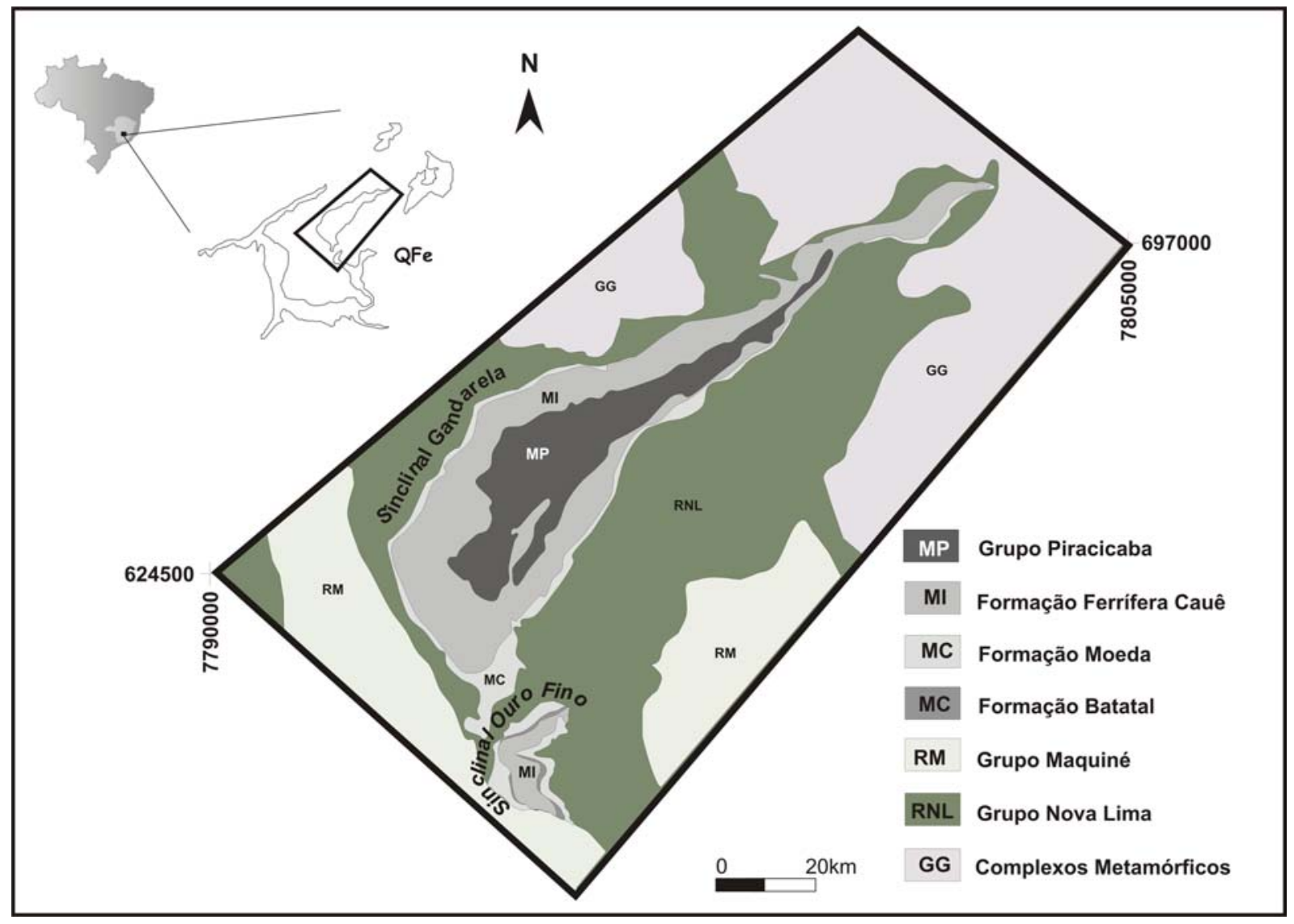

Figura 1 - Mapa de localização do sinclinal Gandarela, Quadrilátero Ferrífero - MG (Dorr, 1969).

inverso formando o sinclinal Gandarela. A fase D3 ocasionou a geração de uma dobra-falha, em geometria cônica, afetando 0 segmento oriental do sinclinal. Desta forma, o sinclinal Gandarela adquiriu uma conformação de dobras reclinadas com eixos de dobras com direção ESE e caimento de 35 (Fig. 2d).

\section{DECONVOLUÇÃO EULER}

0 algoritmo de deconvolução Euler 2D foi desenvolvido com intuito de estimar as profundidades de fontes magnéticas ou de corpos magnéticos. A técnica foi desenvolvida por Thompson (1982) que se baseia na equação diferencial homogênea de Euler, considerando as coordenadas cartesianas $x, y, z$ e a função descrita por $f(x, y, z)$. Seja $f(x, y, z)$ uma função dita homogênea de grau $n$, se:

$$
f(t x, t y, t z)=t^{n} f(x, y, z)
$$

0 plano de observação é dado por $z=0$, positivo para baixo. Por convenção, eixo $x$ na direção norte e $y$ na direção leste.
Satisfazendo a equação diferencial parcial, tem-se:

$$
x \frac{\partial f}{\partial x}+y \frac{\partial f}{\partial y}+z \frac{\partial f}{\partial z}=n f
$$

tendo então,

$$
f(x, y, z)=\frac{G}{r^{N}}
$$

onde $r=\left(x^{2}+y^{2}+z^{2}\right)^{1 / 2}, N=1,2,3, \ldots, G$ é uma constante e não depende de $x, y, z$ e $n=-N$.

Considerando uma fonte pontual como uma massa pontual ou um dipolo magnético localizado no ponto $\left(x_{0}, y_{0}, z_{0}\right)$ relativo ao plano medido, a intensidade do campo magnético total pode ser expressa por:

$$
\Delta T(x, y)=f\left[\left(x-x_{0}\right),\left(y-y_{0}\right), z_{0}\right],
$$

de tal forma que a equação de Euler pode ser descrita na forma:

$$
\begin{aligned}
\left(x-x_{0}\right) \frac{\partial \Delta T}{\partial x} & +\left(y-y_{0}\right) \frac{\partial \Delta T}{\partial y}-z_{0} \frac{\partial \Delta T}{\partial z} \\
& =-N \Delta T(x, y)
\end{aligned}
$$




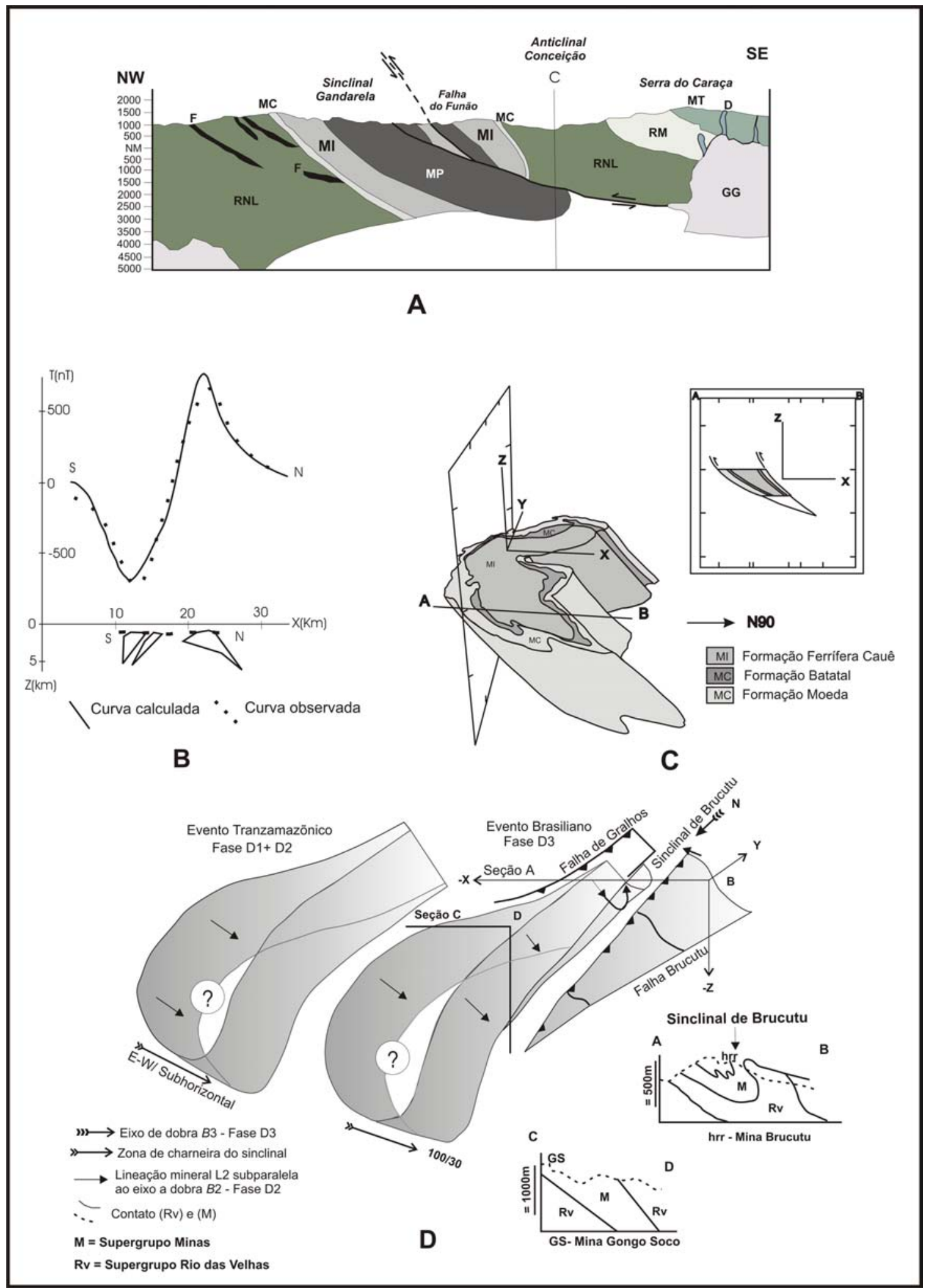

Figura 2 - Modelos evolutivos para a região do sinclinal Gandarela: A) Dorr (1969); B) Padilha (1982); C) Franco (2003); D) Endo et al. (2004). 
onde $N$ é 0 índice estrutural que está vinculado à geometria da fonte causadora da anomalia e interfere diretamente no decaimento do campo magnético relativo ao plano medido de acordo com o modelo assumido (corpos cilíndricos, esféricos, diques finos, etc.) para a fonte magnética, os símbolos são adotados para indicar os índices estruturais utilizados nos perfis de deconvolução Euler 2D.

0 programa EULDEP foi desenvolvido com intuito de estimar profundidades médias de fontes magnéticas. 0 conceito fundamental do programa é baseado na idéia de camadas equivalentes que estão relacionadas aos corpos causadores de anomalias magnéticas com magnetização uniforme.

A distância de cada fonte pode apresentar características dipolares, ou seja, um corpo magnético extrusivo pode ter pólos induzidos positivos na sua superfície superior e pólos induzidos negativos na sua superfície inferior. Em contrapartida, corpos magnéticos intrusivos podem ter pólos induzidos positivos no topo, mas os pólos negativos localizados na base, não contribuem no campo magnético medido e, conseqüentemente podem exibir caráter polar. (Thompson, 1982).

É importante salientar que a deconvolução não produz modelos geológicos através do campo magnético e, sim, tentativas muito restritas a serem aplicadas em algumas situações geológicas (Thompson, 1982). 0 tamanho da janela utilizado deve ser pequeno para englobar apenas uma anomalia, dando maior confiabilidade no resultado. Caso contrário, poderá agrupar duas ou mais anomalias fornecendo resultado incorreto (Durrheim \& Cooper, 1998).

Reid et al. (1990) implementaram algoritmo 2D de Thompson (1982) para a interpretação magnética em três dimensões com base na equação homogênea de Euler descrita na forma:

$$
\begin{aligned}
\left(x-x_{0}\right) \frac{\partial T}{\partial x} & +\left(y-y_{0}\right) \frac{\partial T}{\partial y}+\left(z-z_{0}\right) \frac{\partial T}{\partial z} \\
& =N(B-T),
\end{aligned}
$$

onde $\left(x_{0}, y_{0}, z_{0}\right)$ é a posição da fonte magnética, $T 0$ campo total definido por $(x, y, z), B$ o valor regional do campo total $\mathrm{e}$ $N$ o grau de homogeneidade ou índice estrutural.

A equação de Euler 3D é facilmente aplicada em grids de dados magnetométricos e pode ser processado da seguinte forma:

a) calcular ou medir o gradiente $\frac{\partial T}{\partial x}, \frac{\partial T}{\partial y}, \frac{\partial T}{\partial z}$;

b) utilizar janelas com tamanho $3 \times 3$. A janela de $10 \times 10$ apresenta um bom resultado, mas quanto menor 0 tamanho da janela maior será a resolução dos resultados. Cada janela fornece uma estimativa de localização única da fonte causadora de anomalia magnética. Quando todas as estimativas são colocadas ao longo do perfil, elas tendem a se agrupar em torno do contraste de magnetização.

c) para cada índice estrutural diferente de zero, são usados todos os pontos na janela para resolver a equação Euler para fontes de posição $\left(x_{0}, y_{0}, z_{0}\right)$ e para obter as estimativas de mínimos quadrados, ou seja, o valor background da solução, é possível utilizar a inversão de MorePenrose baseada em mínimos quadrados (Lawson \& Hanson, 1974).

d) para o índice estrutural igual a zero, procede-se de maneira semelhante ao item c, porém a equação a ser utilizada é:

$$
\left(x-x_{0}\right) \frac{\partial T}{\partial x}+\left(y-y_{0}\right) \frac{\partial T}{\partial y}+\left(z-z_{0}\right) \frac{\partial T}{\partial z}=A(7)
$$

sendo $A=2 M t \cos \Psi, M$ a intensidade de magnetização, $t$ a espessura de corpos tabulares $(t \ll$ $\left.z_{0}\right)$ e $\cos \Psi=\left[\cos ^{2} \alpha \cos ^{2} I+\sin ^{2} I\right]^{1 / 2}$, onde $\alpha$ é 0 azimute do perfil e $I$ a inclinação do campo geomagnético.

e) para cada índice estrutural a solução se dá através da visualização em mapas, onde cada solução é representada pelo plano $(x, y)$ usando símbolos proporcionais à profundidade $z$.

\section{METODOLOGIA}

Os dados magnetométricos utilizados pertencem ao Projeto Brasil-Alemanha realizado em Minas Gerais e disponibilizados pela CPRM. Através destes, foi possível gerar mapas de contorno corrigido de IGRF (International Geomagnetic Reference Field) e de redução ao equador magnético.

Foram selecionados dois perfis magnetométricos ao longo da região investigada, sendo o perfil AA' coincidente com a seção geológica de Dorr (1969) (Fig. 2a) e com o perfil de inversão magnetométrica 2D de Padilha (1982) (Fig. 2b).

Para a deconvolução Euler 2D, os perfis foram transformados em arquivos $A S C I /$, os dados inseridos para a geração dos perfis são a intensidade do campo total de 24090nT, a inclinação de $-22,0^{\circ}$ e a declinação de $-17,8^{\circ}$ em relação ao ano do dado coletado (Projeto Brasil-Alemanha) e a altura de vôo de 350m, em média. 0 índice estrutural utilizado para 0 ajuste de contatos foi 1,0 (opção sugerida pelo software utilizado na geração dos perfis) e 0 tamanho da janela para controlar a absorção de fontes magnéticas foi 11. 


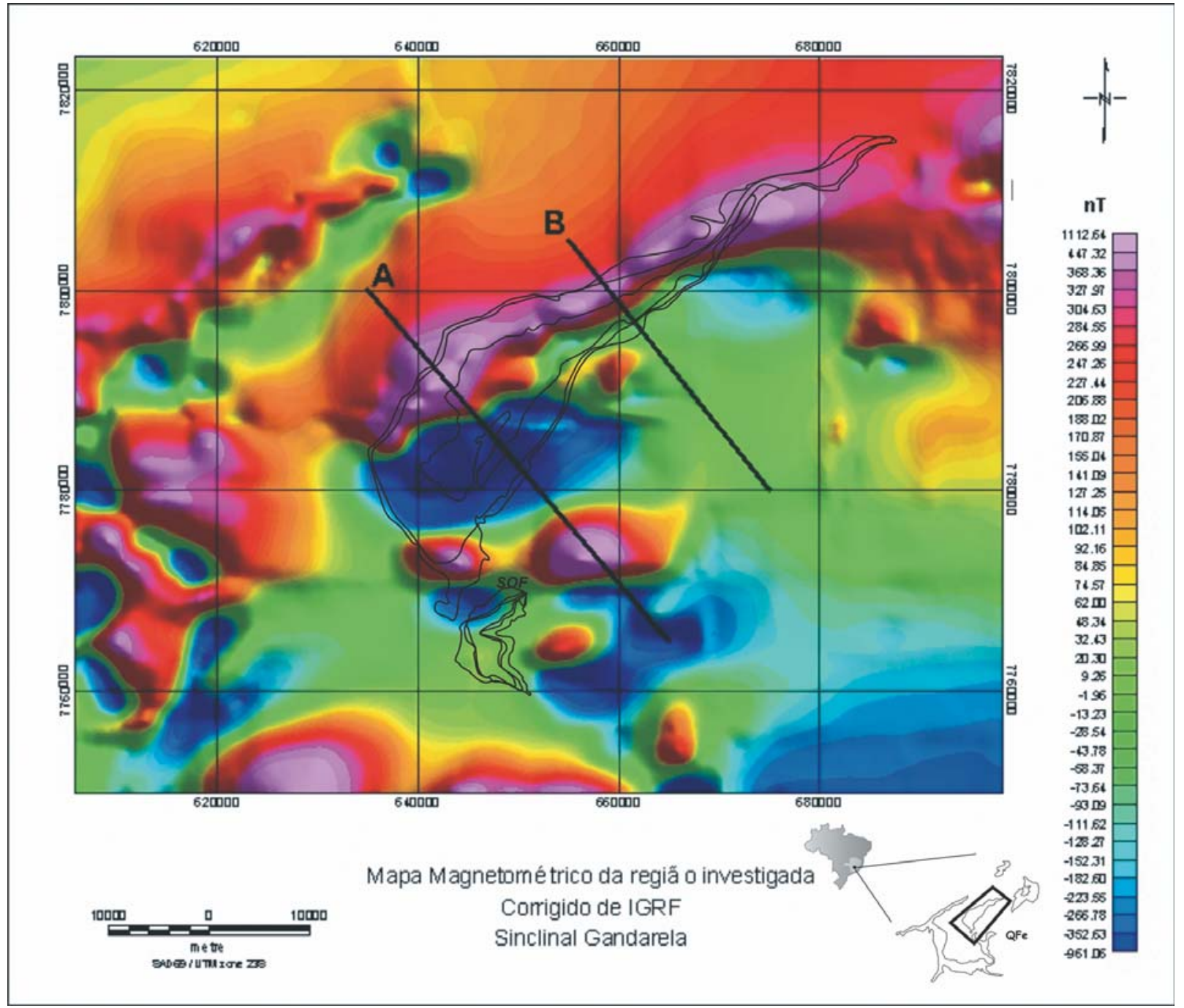

Figura 3 - Mapa magnetométrico corrigido de IGRF, ilustrando os perfis selecionados na região. SOF = sinclinal Ouro Fino.

0 mapa de deconvolução Euler 3D foi gerado a partir do grid de primeira derivada, onde foi possível determinar os intervalos de profundidade para, então, comparar os resultados das deconvoluções com a geologia.

\section{RESULTADOS E DISCUSSÕES}

No mapa magnetométrico corrigido de IGRF, foram observadas anomalias de amplitude positiva nos grupos Nova Lima e Itabira relacionadas às respectivas formações ferríferas. As anomalias de amplitudes intermediárias estão associadas aos grupos Nova Lima, Maquiné e Caraça. Já as anomalias de pouca intensidade magnética correspondem ao Grupo Piracicaba. É possível observar feições estruturais de direção EW na região (Fig. 3).

No mapa de contorno de redução ao equador magnético, as anomalias de amplitude positiva foram realçadas e confirmadas nas formações ferríferas dos grupos Nova Lima e Itabira, destacando-se também as anomalias intermediárias e amplitudes negativas relacionadas às demais unidades. 0 padrão estrutural apresenta-se igualmente acentuado na direção EW (Fig. 4).

Os perfis de deconvolução Euler 2D auxiliaram na caracterização da geometria do sinclinal Gandarela e na determinação das profundidades médias do Grupo Nova Lima e de rochas granito-gnáissicas pertencentes ao Complexo Metamórfico Caeté. No perfil AA', a inclinação do flanco SE do sinclinal Gandarela possui um mergulho mais acentuado em relação ao flanco NW, a interpretação do contato da estrutura Gandarela em profundidade, se baseia a partir de dados de superfície (Fig. 5).

No perfil BB', foi possível estimar as profundidades médias 


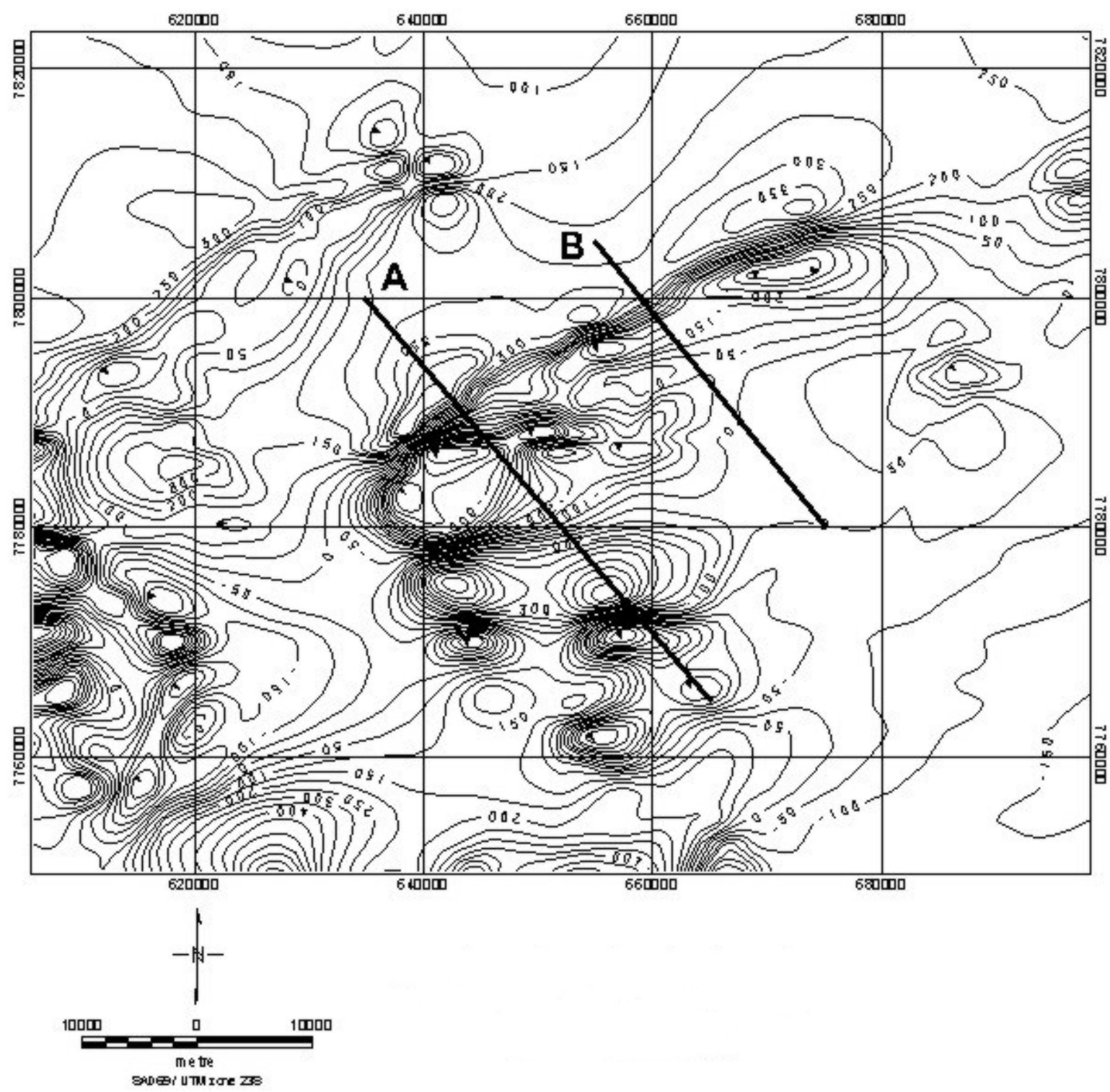

Figura 4 - Mapa de contorno magnetométrico com redução ao equador magnético.

do Grupo Nova Lima em torno de 3500 a 4500 m e caracterizar 0 adelgaçamento tectônico do sinclinal Gandarela em profundidade, obtido por dados de superfície como visto no perfil $\mathrm{AA}^{\prime}$ (Fig. 6).

No mapa de deconvolução Euler 3D, a estrutura do Gandarela alcança profundidades entre 2000 a $3000 \mathrm{~m}$. A profundidade do Grupo Nova Lima varia de 3000 m, no segmento NW, a 4000 m, no segmento SE do sinclinal Gandarela (Fig. 7).

Em relação à geometria do sinclinal Gandarela interpretada nos perfis $\mathrm{AA}^{\prime}$ e $\mathrm{BB}$ ' de deconvolução Euler 2D, notou-se um adelgaçamento tectônico em profundidade, corroborando com os resultados obtidos por Padilha (1982) e Franco (2003).

Oliveira (2005) alcançou resultados similares à dos autores supracitados. Utilizando-se de seções geológicas coincidentes com os perfis magnetométricos, obteve uma interpretação inicial do ajuste de contatos em subsuperfície, pela deconvolução Euler 2D. Em seguida, esse ajuste foi encaminhado para o método de modelagem poligonal 2D, refinando a geometria do sinclinal Gandarela, considerando como parâmetros de controle, a susceptibilidade magnética e a densidade das rochas (Fig. 8). 


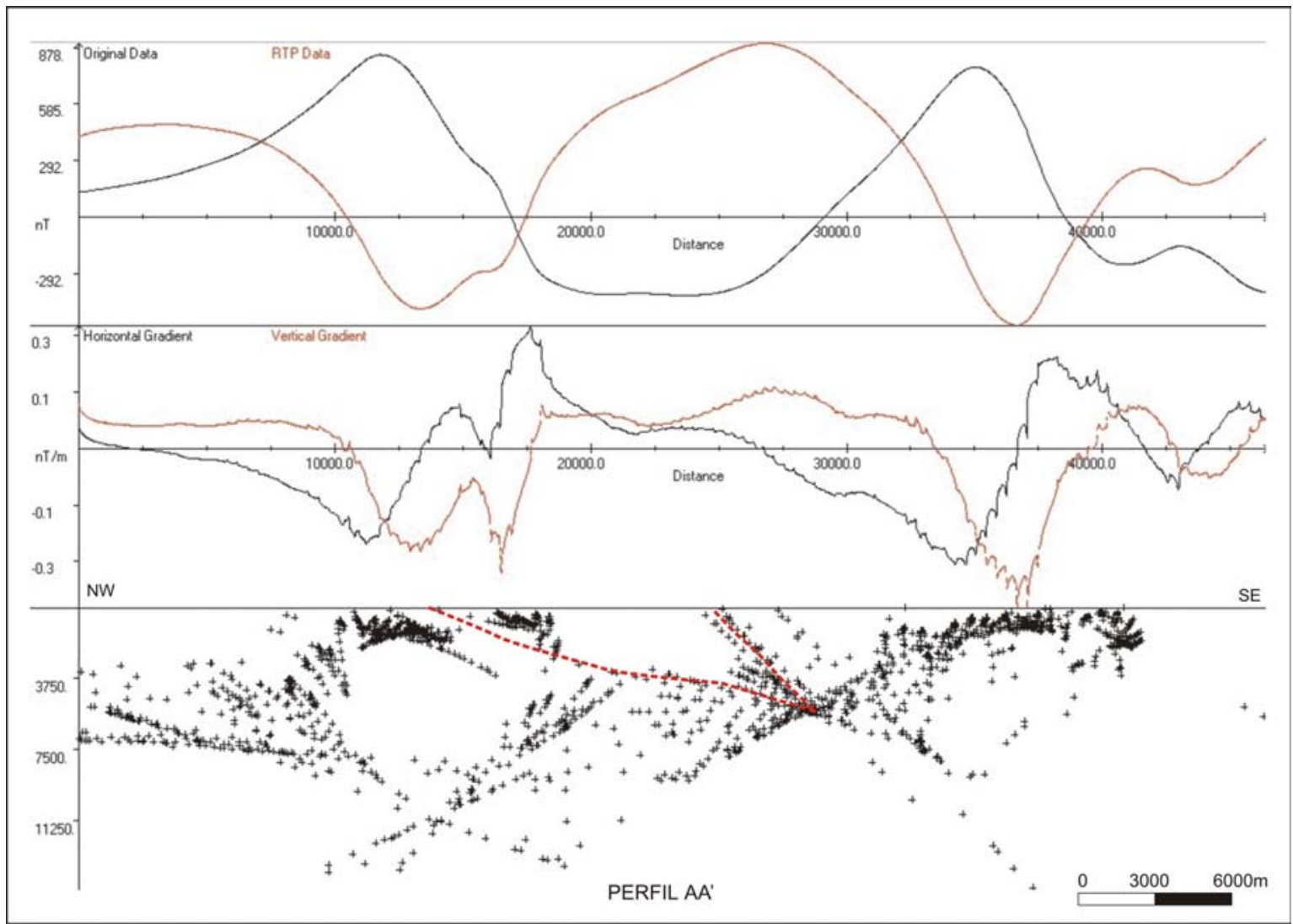

Figura 5 - Perfil AA' de deconvolução Euler 2D na região do Sinclinal Gandarela coincidente com perfis geológico e de inversão magnetométrica de Dorr (1969) e Padilha (1982), respectivamente.

\section{CONCLUSÕES}

0 método de deconvolução de Euler permitiu estimar as profundidades médias das unidades magnéticas que incluem rochas supracrustais e granito-gnáissicas. Aliado ao conhecimento geológico da estrutura modelada foi possível caracterizar a geometria da mesma, apresentando-se similar à modelagem $2 \mathrm{D} \mathrm{e}$ as inversões Linear e não-Linear 2D obtidas por Oliveira (2005), sendo compatível aos modelos existentes (e.g. Padilha, 1982; Franco, 2003; Endo et al., 2004), confirmando 0 adelgaçamento tectônico progressivo tanto da estrutura quanto das camadas, em profundidade.

Em terrenos complexos, como é o caso do Quadrilátero Ferrífero, esta ferramenta mostrou-se bastante viável na determinação do arcabouço estrutural, auxiliando o entendimento da geometria dos corpos magnéticos.

\section{AGRADECIMENTOS}

Os autores agradecem à CPRM pelo fornecimento de dados aerogeofísicos do Projeto Brasil-Alemanha, ao NUPETRO (Fundação
Gorceix/Escola de Minas) pela disponibilidade de computadores e softwares utilizados no decorrer do trabalho e aos revisores pelas correções e sugestões. A primeira autora agradece a CAPES pela concessão da bolsa de mestrado.

\section{REFERÊNCIAS}

ALKMIM FF \& MARSHAK S. 1998. Transamazonian orogeny in the Southern São Francisco craton region, Minas Gerais, Brazil: evidence for Paleoproterozoic collision and collapse in the Quadrilátero Ferrífero. Precamb. Res., 90: 29-58.

BLITZKOW D, GASPARINI P, MANTOVANI MSM \& SÁ NC. 1979. Crustal structures of SE Minas Gerais, Brazil deduced from gravity measurements. Rev. Bras. Geoc., 9: 39-43.

CHAUVET A \& MENEZES M. 1992. Évolution structurale du sud du Craton São Francisco: implications sur les minéralisations auriferes de la région d' Ouro Preto, Brésil. C.R. Acad. Sci. Paris, Série II, 315: 495501.

CHEMALE F-Jr, ROSIĖRE CA \& ENDO I. 1991. Evolução tectônica do 


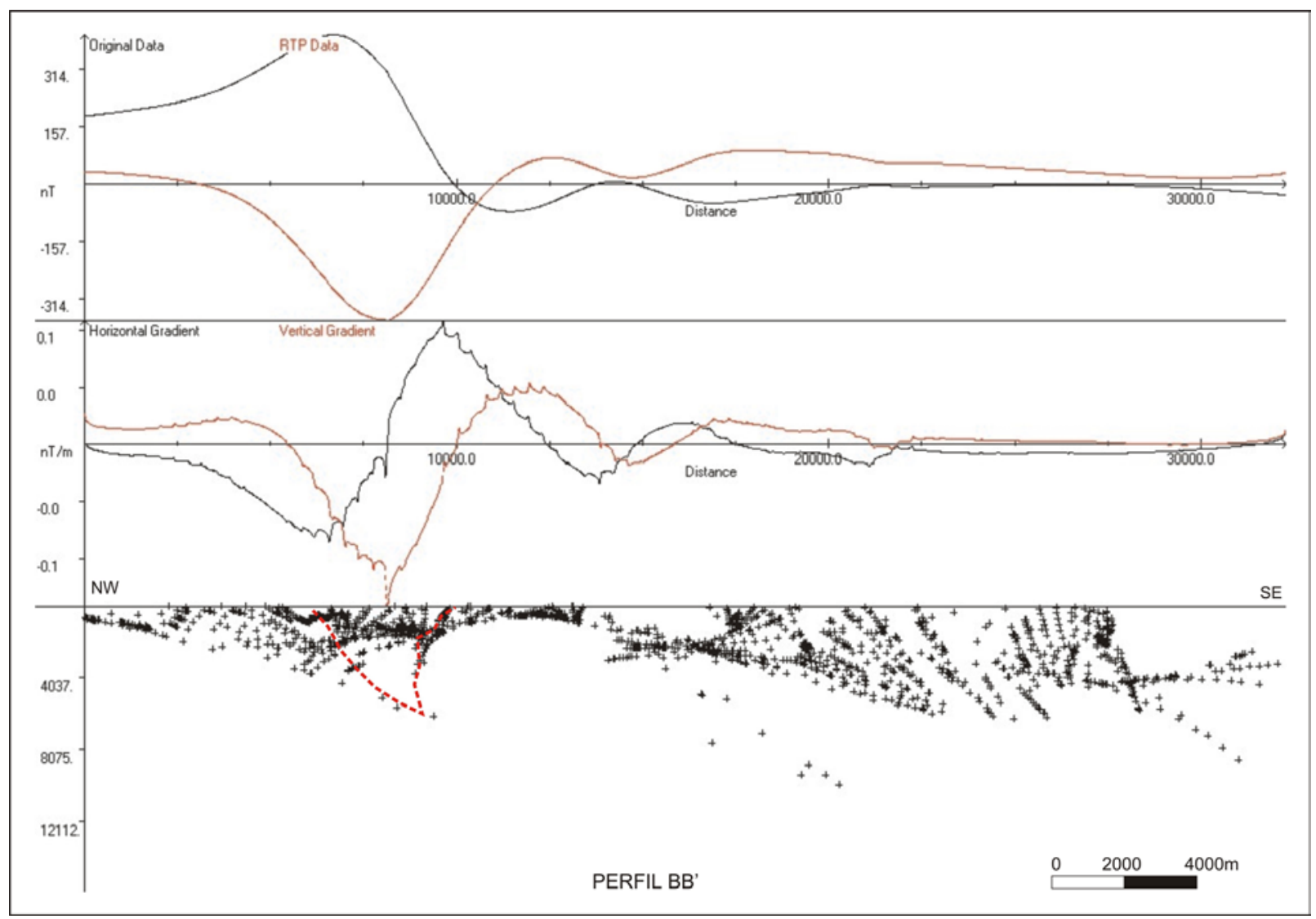

Figura 6 - Perfil BB' de deconvolução Euler 2D na região investigada, confirmando a geometria da estrutura Gandarela definida por Padilha (1982) e Franco (2003).

Quadrilátero Ferrífero, Minas Gerais: Um modelo. Pesquisas, 18(2): 104-127.

CHEMALE F-Jr, ROSIÈRE CA \& ENDO I. 1994. The tectonic evolution of the Quadrilátero Ferrífero, Minas Gerais, Brazil. Precamb. Res., 65: 25-54.

DNPM. DEPARTAMENTO NACIONAL DE PRODUÇÃO MINERAL. 1974. Retrospectivas das atividades de convênio Brasil-Alemanha e trabalhos do Centro de Geofísica Aplicada. Brasília, DNPM, Boletim das Minas e Energia.

DORR JVN. 1969. Physiographic, Stratigraphic and Structural development of the Quadrilátero Ferrífero, Minas Gerais, Brazil. Washington, USGS/DNPM. Prof. Paper 641-A, 110p.

DOSSIN IA, DOSSIN TM, CHAUVET J \& CHEMALE Jr-F. 1992. Tectonique du Protérozoique supériuer au sud-est du Craton São Francisco (Minas Gerais, Brésil), C. R. Acad. Sci. Paris, Série II., 315: 629-636.

DURRHEIM RJ. 1983. Regional-residual separation and automatic interpretation of aeromagnetic data. M.Sc. Thesis, University of Pretoria, South Africa, 117pp.
DURRHEIM RJ \& COOPER GRJ. 1998. Euldep: A program for the Euler deconvolution of magnetic and gravity data. Computers \& Geosciences, 24(6): 545-550.

ENDO I. 1997. Regimes tectônicos do Arqueano e Proterozóico no interior da Placa Sanfranciscana: Quadrilátero Ferrífero e áreas adjacentes, Minas Gerais. Instituto de Geociências, Universidade de São Paulo, São Paulo, Tese de Doutoramento, 243p.

ENDO I \& ROCHA FILHO OG. 2003. Estratigrafia, arcabouço estrutural e controle da mineralização ferrífera da mina de Gongo Soco, MG. 48p. Relatório Interno.

ENDO I, SILVA LG \& ZAVAGLIA G. 2004. Estratigrafia e arcabouço estrutural do Complexo Ferrífero Brucutú-Dois Irmãos - segmento NE do sinclinal Gandarela. 20p. Relatório Interno.

FRANCO ASP. 2003. Geometria e evolução tectônica do Sinclinal Ouro Fino e implicações para a mineralização ferrífera - Quadrilátero Ferrífero, MG. Departamento de Geologia, Escola de Minas da Universidade Federal de Ouro Preto, Ouro Preto, Dissertação de Mestrado, $101 \mathrm{p}$.

GASPARINI P, MANTOVANI MSM, CORRADO G \& RAPOLLA A. 1979. Depth of Curie temperature in continental shields: a compositional boundary? Nature, 278: 845-846. 


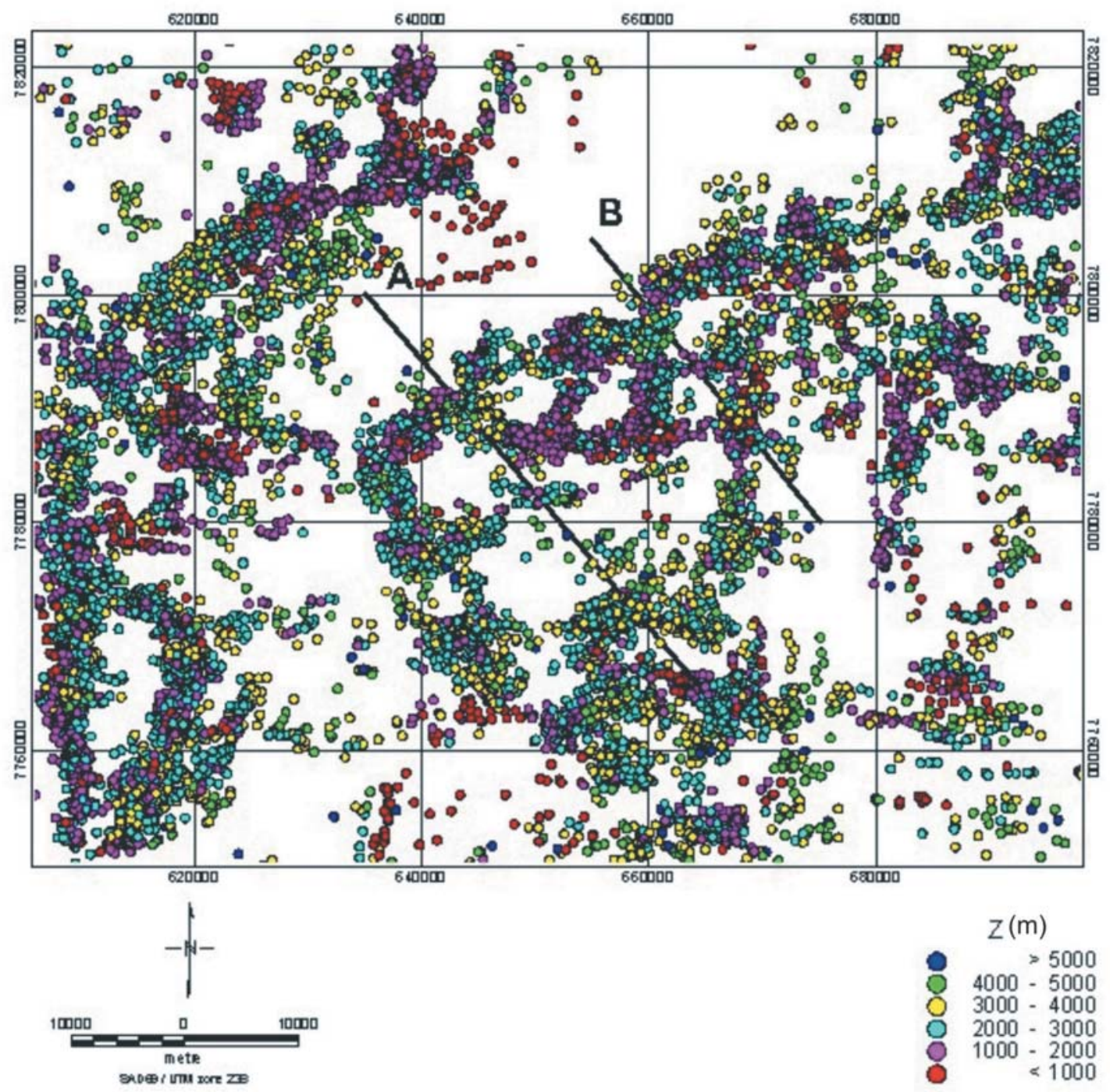

Figura 7 - Mapa de deconvolução Euler 3D da região investigada.

HARTMAN RR, TESKEY DJ \& FRIEDBERG JL. 1971. A system for rapid digital aeromagnetic interpretation. Geophysics, 36(5): 891-918.

HIPPERTT JF \& DAVIS B. 2000. Dome emplacement and formation of kilometer-scale-synclines in a granite-greenstone terrain (Quadrilátero Ferrífero, southeastern Brazil). Precamb. Res., 102: 99-121.

H00D PJ. 1965. Gradient measurements in aeromagnetic surveying. Geophysics, 30(1): 891-902.

LAWSON CL \& HANSON RJ. 1974. Solving least squares problems: Prentice-Hall Inc.

MAXWEL CH. 1960. Mapa Geológico da Quadrícula Capanema, Minas Gerais. Washington, USGS/DNPM. Prof. Paper 341J, 73 p.

OLIVEIRA NV. 2005. Modelagem e Inversão 2D de dados magne- tométricos aplicados na caracterização geométrica do sinclinal Gandarela e Homoclinal Curral, Quadrilátero Ferrífero, MG. Dissertação de Mestrado, Departamento de Geologia/Escola de Minas, Universidade Federal de Ouro Preto, $124 p$.

O' ROURKE JE. 1957. The Stratigraphy of the metamorphic rocks of the Rio de Pedras and Gandarela Quadrangles, Minas Gerais, Brazil. University of Wisconsin, Wisconsin, Ph.D. Thesis, $106 \mathrm{p}$.

PADILHA AL. 1982. Implementação da Metodologia para Interpretação Magnética e sua Aplicação aos dados do Quadrilátero Ferrífero (MG). Instituto de Astronomia e Geofísica, Universidade de São Paulo, dissertação de mestrado, 147 p.

PETERS LEO J. 1949. The direct approach to magnetic interpretation and its practical application: Geophysics, 14: 290-320. 


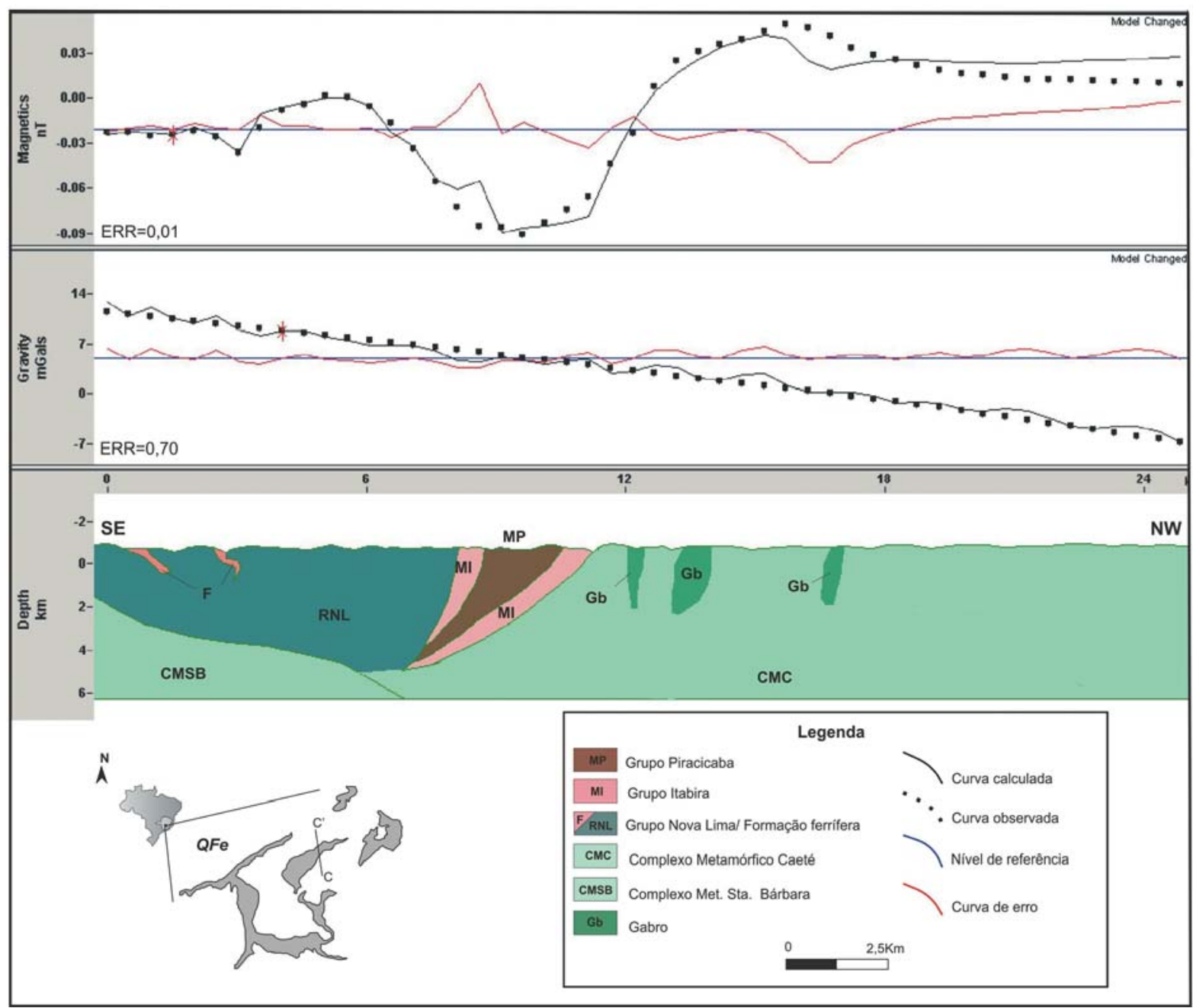

Figura 8 - Método Poligonal de Modelagem 2D da região do sinclinal Gandarela, realizada a partir da integração da seção geológica ao perfil de deconvolução Euler 2D.

REID AB, ALLSOP JM, GRANSER H, MILLET AJ \& SOMERTON IW. 1990. Magnetic interpretation in three dimensions using Euler deconvolution. Geophysics, 55: 80-91.

ROLIM SBA. 2001. Resposta Geofísica dos Depósitos de Ouro da Porção Central do Quadrilátero Ferrífero, MG. Instituto de Geociências, Universidade Estadual de Campinas, Tese de Doutoramento, 290 p.

SILVA AM. 1999. Integração de dados geológicos e geofísicos utilizandose uma nova técnica estatística para seleção de alvos para exploração mineral, aplicada ao Greenstone Belt Rio das Velhas, Quadrilátero Ferrífero. Universidade de Brasília, Tese de Doutoramento, 195 p.
TAVARES P. 1998. Identificação de Áreas Potencialmente Favoráveis à Mineralização Aurífera, a partir de Dados Estruturais e Aerogeofísicos aplicados a Terrenos do tipo "Greenstone Belt": 0 Caso da Folha Caeté, QF, MG. Departamento de Geologia, Escola de Minas da Universidade Federal de Ouro Preto, MG, Tese de Doutoramento, 209 p.

THOMPSON DT. 1982. EULDPH. A new technique for making computerassisted depth estimates from magnetic data. Geophysics, 47: 31-37.

WERNER S. 1953. Interpretation of magnetic anomalies at sheet-like bodies: Sver. Geol. Undersok, ser. C. C. Arsbok, 43 (6). 


\section{NOTAS SOBRE OS AUTORES}

Natália Valadares de Oliveira é engenheira geóloga pela Universidade Federal de Ouro Preto (2002), atuou como consultora nas áreas de petróleo e minério de ferro, envolvendo perfilagem, magnetometria e integração geologia/sísmica de reflexão, pela Fundação Gorceix/NUPETRO (2002/2003). Mestre em Ciências da Terra, com ênfase em Geofísica, pela Universidade Federal de Ouro Preto (2005). Atualmente é doutoranda na área de Geologia Estrutural/Tectônica com ênfase em Geofísica pela Universidade Federal de Ouro Preto. Áreas de interesse: Métodos Potenciais, Geofísica de Prospecção e Geotectônica.

Issamu Endo é graduado em Engenharia Geológica (1980) e Mestre em Geologia Estrutural e Tectônica (1988) pela Universidade Federal de Ouro Preto. Doutor em Geotectônica pelo Instituto de Geociências da Universidade de São Paulo (1997). Professor Adjunto do Departamento de Geologia da Escola de Minas da UFOP desde 1997 onde leciona na graduação e pós-graduação as disciplinas de Geologia Estrutural, Análise Estrutural e Trabalho Geológico. Áreas de interesse: Estratigrafia e Tectônica de Cinturões Pré-Cambrianos.

Luiz Gabriel Souza de Oliveira é geólogo pela Universidade de Brasília (2000). Mestre em Ciências da Terra, com ênfase em Tectonofísica, pela Universidade Federal de Ouro Preto (2003). Atualmente é professor substituto do Departamento de Física e doutorando do PPG-ECRN do Departamento de Geologia, ambos pertencentes à Universidade Federal de Ouro Preto. Suas áreas de interesse envolvem Métodos Potenciais, Tectonofísica e Física de Interiores Planetários. 